\title{
Quaderni
}

QUADERN I Communication, technologies, pouvoir

76 | Automne 2011

Les promesses de la biodiversité

\section{La rationalisation économique du rapport à la biodiversité : éléments d'ethnographie}

Michel Daccache

\section{(2) OpenEdition}

Journals

Édition électronique

URL : http://journals.openedition.org/quaderni/126

DOI : 10.4000/quaderni.126

ISSN : 2105-2956

Éditeur

Les éditions de la Maison des sciences de l'Homme

Édition imprimée

Date de publication : 1 septembre 2011

Pagination : 53-65

Référence électronique

Michel Daccache, «La rationalisation économique du rapport à la biodiversité : éléments

d'ethnographie », Quaderni [En ligne], 76 | Automne 2011, mis en ligne le 01 septembre 2014, consulté

le 19 avril 2019. URL : http://journals.openedition.org/quaderni/126 ; DOI : 10.4000/quaderni.126 


\section{$D$ ossier}

\section{la rationalisation}

économique du rapport à la

biodiversité :

\section{éléments}

\section{d'ethnographie}

\section{Michel Daccache}

Post-doctorant, CEMAGREF (UR DTGR)
L' " érosion de la biodiversité » a fait l'objet, au cours des dernières années, d'un processus d'alerte et de mise à l'agenda scientifique, politique et médiatique ${ }^{1}$. Cette dynamique a conduit à l'émergence d'instances de gouvernance - de différentes natures et de différents niveaux (local, national et international) - qui ont pour enjeux la mesure de cette crise et l'élaboration de technologies de maitrise. Parmi celles-ci, le recours aux mécanismes du marché semble l'une des pistes privilégiées et en tous cas des plus discutées.

L'idée d'agir pour la biodiversité à travers sa mise en marché n'est pas neuve (McAfeen 1999; Aubertin, Boisvert, \& Pinton, 2007; Blandin, 2009). Son importance s'est cependant accrue au cours des dernières années, à mesure que l'alerte devenait plus pressante. La littérature consacrée à cette problématique en témoigne. Toujours plus abondante, elle se polarise selon des principes qui tendent eux-mêmes à se diversifier : unités de mesure et méthodologies retenues, rapports à l'outil monétaire, enjeux éthiques ou politiques, etc. ${ }^{2}$. Certaines revues (Ecological Economics, Conservation Biology, Science...) sont devenues les arènes d'un débat qui tend à se radicaliser ${ }^{3}$. Les disciplines participant de cette controverse sont nombreuses : sciences de la nature (biologie, écologie, génétique...), économie, philosophie, anthropologie... Les critiques portant sur le principe même de la mise en marché de la biodiversité, même si elles sont nombreuses, ne seront pas détaillées, dans la mesure où le pré-requis fondamental des instances dont il est ici question est la croyance en les vertus du marché. À titre d'exemple, on peut cependant évoquer certains textes assimilant les SE, dans leur essence même, à un « fétichisme de la marchandise » (Kosoy \& 
Corbera, 2010) ou encore des travaux d'ordre philosophique soumettant ces mêmes SE au crible du jugement éthique (Maris \& Revérêt, 2009). Quoiqu'il en soit, peu de lectures proprement sociologiques de la dynamique de mise en marché de la biodiversité ont été proposées à ce jour, les analyses touchant aux outils eux-mêmes - à leurs propriétés, à leur valeur, à leur efficacité - plutôt qu'à leurs modes de production, de diffusion ou à leurs usages ${ }^{4}$.

L'objectif du texte suivant est d'envisager les instruments de rationalisation économique $d u$ rapport à la biodiversité non comme des objets figés justiciables d'une lecture « internaliste » ou comme des outils passibles d'une appréciation purement technique, mais comme des productions sociales, ayant une genèse, une histoire, des logiques de circulation, des usages et des fonctions multiples. Afin d'en rendre compte, nous évoquerons d'abord l'histoire de cette mise en économie de la biodiversité à travers un retour sur l'émergence d'instances et d'instruments spécifiques. Nous nous pencherons ensuite sur les caractéristiques de ces espaces - au sein desquels se redéfinissent les relations savoirs/pouvoirs en nous appuyant sur le cas français. Nous nous interrogerons enfin sur le fonctionnement de ces instances en insistant d'une part sur la dynamique de coproduction dont elles sont le lieu et d'autre part sur les conditions sociales d'apparition de ces nouveaux collectifs 5 .

\section{La mise en économie de la biodiversité : une dynamique institutionnelle et instrumentale}

L'histoire des tentatives de prise en charge des enjeux environnementaux par l'économie est ancienne. L'ambition n'est pas ici d'en rendre entièrement compte et encore moins de faire le récit de la théorie économique en la matière. Il s'agit plutôt d'évoquer un tournant dans la gestion de la diversité biologique, marquée par la présence croissante du registre économique associée à la multiplication d'instances participant de ce que l'on pourrait appeler une gouvernementalité de type néolibéral, au sens attribué à cette expression par M. Foucault (Foucault, 2001, 2004). Par gouvernementalité, il faut entendre une « conduite des conduites $»^{6}$, le « nom de l'articulation entre deux types de moyens, des « technologies de pouvoir » et des « techniques de soi » ou, pour le dire autrement, entre des dispositifs et des dispositions entretenues » (Jeanpierre, 2006, p. 7). Quant à l'adjectif néolibéral, il doit ici être compris comme renvoyant à une « raison du moindre État 》 (Foucault, 2004, p.30) associée à la concurrence généralisée comme principe de régulation et à l'instauration du marché comme lieu de véridiction?

\section{Un dispositif institutionnel...}

Objet de cette évolution, la biodiversité en est également indicateur et opérateur. En effet, le terme de biodiversité apparaît dans les années 1980, à un moment où les instances de pilotage environnementales semblent se tourner plus franchement vers le marché et l'entreprise privée. Ce changement de paradigme s'observe notamment au niveau de l'UICN (Union Internationale pour la Conservation de la Nature), l'une des institutions centrales dans le domaine. Alors que les années 1950-60 sont marquées par l'approche préservationniste et les années 1960-80 par l'utilitarisme, c'est un paradigme économique 
et néolibéral qui prend ensuite le pas, ce dont la publication par l'UICN de l'ouvrage de Mc Neely, Economics and Biological Diversity (1988) témoigne parfaitement ${ }^{8}$. Le Sommet de la Terre de Rio (1992) accentue cette tendance. C'est en effet à l'occasion ou dans le sillage de celui-ci que sont créées nombre d'instances visant à représenter/mobiliser les acteurs privés. Parmi celles-ci, des associations d'entreprises telles que le WBCSD (World Business Council for Sustainable Development) et, pour la France, EPE (Entreprises pour l'Environnement), créées en 1992. Dans un registre légèrement différent, on trouve entre autres Orée, association «multiacteurs » regroupant entreprises, collectivités territoriales et associations écologistes (1992). Des programmes de recherche de grande ampleur visant à faire le jour sur la dimension économique des interactions entre activités humaines et biodiversité sont également lancés : le MEA (Millenium Ecosystem Assesment) commandé par le Président des Nations-Unies (2001-2005); le programme "Business and Biodiversity " de l'UICN (2003) ; le programme « Business and Biodiversity Offset » (2009) qui associe des entreprises à des ONG (Organisations NonGouvernementales); et surtout le TEEB (The Economics of Ecosystems and Biodiversity), qui est une émanation du PNUE, le Programme des Nations Unies pour l'Environnement (20082010). Des instances internationales d'incitation à la recherche telles que l'EPBRS (European Platform for Biodiversity Research Strategy), créée en 1999, s'orientent vers cette problématique. L'ouverture aux entreprises s'accentue : en 2006, la huitième conférence des parties ( $« \mathrm{COP} 8 »)$ de la CDB (Convention sur la Diversité Biologique) adopte à Curitiba une décision (VIII/17) visant la contribution du secteur privé aux objectifs de la convention; au même moment l'UICN, ainsi que de grandes ONG (World Wide Fund for Nature, WWF ; Conservation International) multiplient partenariats et conventions-cadres avec des entreprises (Véolia, EDF, Yves Rocher...). Réciproquement les entreprises, notamment à travers leurs fondations (Fondations Hermès, Total...) se tournent vers la biodiversité. L'année 2010, déclarée année internationale de la biodiversité par l'ONU, renforce encore ce mouvement : les travaux du TEEB sont publiés sous forme d'un rapport (le « Rapport Sukhdev ») ; la dixième conférence des parties ( «COP $10 »)$ de la Conférence sur la Diversité Biologique, à Nagoya, met la question de l'économie de la biodiversité au centre des débats ; la naissance de l'IPBES, la Plateforme Intergouvernementale sur la Biodiversité et les Services Ecosystémiques faisant du recours au marché un instrument central, est entérinée.

\section{... et instrumental}

En étroite relation avec cette dynamique d'institutionnalisation, les instruments ${ }^{9}$ de rationalisation économique du rapport à la biodiversité se développent et se raffinent ${ }^{10}$. Comme pour le climat (avec le bilan carbone et l'établissement d'un marché de droits), il s'agit d'élaborer une unité de mesure à laquelle associer des mécanismes de régulation. Cette unité de mesure (ou plutôt de cadrage permettant des mesures), ce sont les Services Ecosystémiques (notés «SE »), définis comme les services que les écosystèmes rendent aux humains (ou qui sont prélevés par ce dernier). Quatre types de services sont classiquement identifiés depuis le MEA (de production, de régulation, de support et culturels) auxquels vont 
pouvoir être attribués une valeur, un prix ou un coût, permettant la mise en place de différents modes de régulation (marchés de droits, certifications, subventions, paiements pour services écosystémiques, fiscalité écologique, compensations, etc. $)^{11}$. La notion de SE serait progressivement apparue dans les années 1970-1980, au moment même où se constitue un champ de l'économie de l'environnement. C'est dans un rapport publié par le MIT (Massachusetts Institute of Technology) en 1970 intitulé Study of Critical Environmental Problem que l'on trouverait la première énumération de ces services ${ }^{12}$. L'article fondateur de l'approche en termes de SE reste cependant celui de Costanza et de ses collègues (Constanza, 1997) qui, bien qu'il ait été suivi d'une controverse qui n'a pas tari, a permis la cristallisation et la médiatisation du concept. La réflexion sur les SE avance d'autant plus dans les années 2000 que nombre d'ONG, instances politiques, économiques ou autres (International Institute for Environment and Development, IIED ; Katoomba Group ; Forest Trends ; Département Environnement de la Banque Mondial...) semblent y voir un recours crédible et encouragent les recherches et les scientifiques (Harold A. Mooney ${ }^{13}$, Rudolf De Groot ${ }^{14} .$. ) allant en ce sens. Cette période est donc marquée par des publications clés visant à améliorer cet instrument ${ }^{15}$. Mais surtout, c'est en 2001 que débute le MEA. Programme émanant de l'ONU, en partie financé par le secteur privé (via le WBCSD et la Fondation Avina notamment), il mobilise plus d'un millier d'experts de par le monde dans le but de rendre compte des services rendus par la nature ${ }^{16}$. Présentés en 2005, ses résultats concluent à une sous-estimation systématique de ces services liée à l'absence de quantification monétaire. Ainsi, les travaux du MEA légitiment et encouragent de telles recherches : la référence aux SE et aux mécanismes de régulation qui leur sont associés, même si elle continue d'essuyer des critiques d'ordre éthique ${ }^{17}$ ou méthodologique ${ }^{18}$, a non seulement voix au chapitre, mais devient incontournable.

\section{Des espaces au sein desquels se redéfinissent les relations savoirs/pouvoirs}

\section{Des lieux tournés vers l'opérationnalisation...}

C'est également au sein ou autour de ces espaces qu'ont lieu les essais d'opérationnalisation de ces instruments. En France notamment, des études sont réalisées sur le sujet, alors qu'institutions et mécanismes ad hoc continuent à se mettre en place. En 2009, le CAS (Centre d'Analyse Stratégique) publie un rapport portant sur « L'approche économique de la biodiversité et des services liés aux écosystèmes » (plus connu sous le nom de « rapport Chevassus-au-Louis ») ${ }^{19}$. Ce rapport préconise notamment de distinguer biodiversités remarquable et ordinaire et de n'intégrer que la seconde aux mécanismes du marché et ce non en lui attribuant un prix, mais en évaluant le coût lié à une cessation de service. Le rapport insiste également sur la nécessité de réguler ces échanges éventuels. De nouvelles instances sont par ailleurs créées, comme CDC Biodiversité, filiale de la Caisse des Dépôts et Consignations intervenant auprès des entreprises pour les assister dans leurs actions « biodiversité » (valorisation, restauration, compensation...). Née en 2008, elle établit une convention-cadre avec le MEEDDM (Ministère de l'Écologie, de l'Énergie, du Développement Durable et de la Mer) pour la période 2010-2013. On pense également 
à NatureParif (2008), agence régionale réunissant différents types d'acteurs autour des enjeux de biodiversité en Île-de-France. De son côté l'AFEP (Association Française des Entreprises Privées) - qui réunit pratiquement tous les dirigeants du CAC40 - soutient des actions dans le domaine.

\section{... associant différents groupes vers un même objectif}

En proportions différentes, divers univers sociaux et divers types d'agents - chercheurs, administrateurs de recherche, cadres d'entreprise, militants écologistes, hauts-fonctionnaires, élus, étudiants, etc. - sont mis en continuité au travers d'espaces et de réseaux tendant à composer des communautés de recherche plus ou moins homogènes, solides, durables, actives... L'une des modalités les plus significatives de cette nouvelle économie des relations savoirs/pouvoirs, est le groupe de travail (noté « GT »). Dans le cadre de la FRB (Fondation pour la Recherche sur la Biodiversité) en 2006 et du Comité Français de l'UICN en 2009 ont ainsi été lancés des GT «Entreprises et Biodiversité ». Forme empruntée au monde de l'entreprise, le GT est représentatif d'une gouvernementalité de type néolibérale. En partie extérieure à l'État, elle ne vise pas directement la Loi : son principe est celui d'une objectivation progressive de normes de comportement au travers de discussions et de « retours d'expérience ». Ce sont aussi des standards éthiques (le vocabulaire des « bonnes » ou des «meilleures pratiques » est là pour le rappeler) qui sont véhiculés. Le GT fonctionne ainsi moins sur le mode de l'injonction que de la stimulation, de la sanction que de l'incitation, de la mise en ordre que de la mise en marche ${ }^{20}$. Ces collectifs s'organisent selon des principes et des modes de fonctionnement communs. Ils naissent généralement de la pérennisation de rencontres, colloques, journées d'études, etc. (ex. : Conférence « Biodiversité », EPE, 2004 ; $1^{\text {er }}$ Colloque Orée « Des experts légitimes pour un vrai débat », 2005 ; « La biodiversité, enjeu écologique, enjeu économique », EPE, 2007). Les thèmes retenus relèvent soit du questionnement pratique (ex. : « Biodiversité et carrières », EPE, 2007) soit de la synthèse à destination des praticiens (ex. : Symposium Orée « Biodiversité et climat avant et après Copenhague », 2009). Le but y est à la fois d'objectiver, de concentrer et de routiniser des pratiques déjà existantes et de produire de nouveaux instruments. Les « cas » étudiés sont rapprochés par le biais d'outils de mise en équivalence (tables, matrices...) et font l'objet de présentations synthétiques ou synoptiques (schémas, diagrammes, PowerPoint...). Mais ces espaces sont aussi les témoins d'une production active d'instruments de gouvernance (ex. : l'Indicateur d'interdépendance Entreprises/ Biodiversité proposé par Orée ${ }^{21}$ ) à partir desquels peuvent être conduits enquêtes, audits ou études (ex. : les « audits biodiversité » proposés par la société Gondwana ; enquête « Les interactions entre les entreprises et la biodiversité », réalisée par l'institut Inspire auprès de 5000 entreprises et présentée en novembre 2010 au salon Pollutec de Lyon) venant en aide à la décision. Il s'agit ensuite de tirer des enseignements généraux matérialisés sous forme d'équipements stables : sites internet, manuels, guides et autres vademecum (ex. : " La biodiversité, un atout pour vos sites entreprises », Guide EPE/Orée/UICN, 2006 ; «Intégrer la biodiversité dans les stratégies d'entreprises», Guide Orée/FRB, 2008 ; «Entreprises, biodiversité et services écosystémiques. 
Quelles interactions et stratégies ? Quelles comptabilités ? », thèse AgroParisTech, Joël Houdet, 2010). Ces structures dédiées servent également de support à l'organisation d'évènements (ex. : «Biodiversité: les entreprises et les scientifiques dialoguent», Journée Orée/ FRB, 2008 ; «Les indicateurs de la biodiversité », rencontre-débat Orée/Natureparif, 2009) et à la distribution d'honneurs (ex. : Remise du prix «Biodiversité et Entreprise», Orée, salon Pollutec de Lyon, 2010).

\section{Un mode de fonctionnement relevant de la «coproduction"}

\section{L'interpellation de la science}

Dans ces espaces, la mise en relation des différentes catégories d'agents s'opère par incitations réciproques et se traduit sous forme de circuits de légitimation croisés. L'interpellation des chercheurs s'effectue via des appels d'offre (Appel à projets de recherche pan-européens sur la biodiversité et les services écosystémiques, FRB/ Biodiversa, 2010 ; cofinancement Véolia-État, sous forme de «Cifre », d'une thèse entre 2008 et 2010 dans le cadre du GT Orée avec pour but d'élaborer une approche comptable incluant une mesure physique et monétaire des flux d'entrée et de sortie de la biodiversité). De telles sollicitations ont d'autant plus de chance de rencontrer d'écho que les instances dont elles émanent se proposent non seulement de financer les recherches, mais de les encadrer, les pourvoir en cas, les publiciser, les valoriser... Elles peuvent éventuellement offrir des positions (professionnelles, académiques...), des postes à charge honorifique (comités scientifiques, d'organisation, de rédaction...), des titres (distinctions diverses : prix, bourses, chaires, etc.). L'emboîtement des niveaux national et international permet également la circulation des travaux et, avec eux, des schèmes de pensée et d'action. Par exemple, les recherches menées au sein d'Orée s'articulent à celles du WBCSD et du WRI, le World Resource Institute. Leurs résultats sont intégrés au rapport du CAS et ont été présentés lors de la conférence des parties de la CDB à Nagoya. Par ailleurs, les textes produits dans le cadre du GT, traduits en anglais, sont autant de contributions au TEEB.

Dans de tels lieux, les chercheurs assument un rôle d'encadrement intellectuel et éthique et se livrent à un double exercice de diffusion de savoirs scientifiques et de mise en forme savante de savoirs profanes. Procédant à une sorte de maïeutique collective, ils encouragent à une réflexivité orientée selon l'enjeu et la problématique du moment. L'incitation prend ici la forme de l'aiguillon qui pousse chacun à dire sa vérité : cadres d'entreprises, militants, usagers, etc. se trouvent transformés en détenteurs d'un savoir qui se doit d'être révélé. Pourvoyeurs de concepts et de rationalisations (au double sens wébérien et freudien du terme), les chercheurs contribuent à une montée en généralité d'expériences singulières et isolées, garantie de la transposabilité2 ${ }^{22}$ des schèmes cognitifs et pratiques qui y sont contenus. Les différentes disciplines sont mobilisées à des degrés et des intensités variées. Si la science économique est la mieux à même de se couler dans des problématiques forgées par des acteurs économiques pour répondre à des problèmes économiques, sciences naturelles (notamment l'écologie) et sciences humaines et sociales (sociologie et anthropologie) ont aussi une place, les premières permettant de fonder 
ces nouveaux instruments économiques dans la nature naturelle, les secondes - au premier rang desquelles la philosophie et l'anthropologie - leur offrant un fondement dans la nature humaine. Accédant à l'universalité par différentes voies, les visions du monde social et naturel investies dans de tels instruments sont d'autant plus légitimes que les circuits de légitimation sont longs ou peu lisibles ${ }^{23}$.

\section{La logique des affinités}

Sur le plan du sens vécu, la rencontre entre ces différents groupes n'apparaît nécessaire que dans la mesure où la situation de crise et d'urgence la rend exigible. Le recours à l'expertise scientifique se donne ainsi à voir comme une nécessité logique et un impératif moral. L'engagement des chercheurs se vit quant à lui sur le mode d'une adaptation plus ou moins enthousiaste au principe de réalité ("le reste a échoué»; "l'économie de marché domine »; "c'est une manière de concilier l'homme et l'environnement »...) ou d'une célébration plus franche des vertus du marché opposées aux lourdeurs étatiques («les entreprises, elles, prennent conscience... »; "l'innovation a lieu du côté des entreprises»; «les décisions publiques sont irrationnelles»...). Le rapprochement entre différentes populations peut également se vivre comme le produit de choix fondés sur la reconnaissance spontanée et réciproque de compétence, d'intelligence, d'ouverture. Ces affinités vécues comme électives n'en demeurent pas moins bien fondées, ce que tend à révéler la connaissance des propriétés sociales des agents. En se basant sur les premiers résultats de l'enquête sociologique, on peut en effet émettre comme hypothèse forte que les instances de rationalisation économique du rapport à la biodiversité sont le produit de la rencontre entre des groupes aux trajectoires et aux caractéristiques sociales hybrides. D'une part, on trouve des cadres supérieurs fortement dotés en capital culturel (diplômes de grandes écoles, notamment d'ingénieurs). Leurs compétences techniques s'assortissent souvent de compétences scientifiques ou culturelles (DEA ou Doctorat de Sociologie, Agronomie, Économie...). Ils occupent généralement au sein de l'entreprise des positions récentes, à forte charge intellectuelle et éthique : responsables biodiversité ou développement durable, chargés de mission environnement, etc. Fonctions auxquelles ils ont d'autant plus besoin de trouver un ancrage savant qu'ils en sont parfois les premiers titulaires. Ils incarnent des idéaux «nouveaux » (entreprise citoyenne, développement durable, dirigeant éthique... $)^{24}$. «Citoyens concernés », ils préfèrent les grandes $\mathrm{ONG}$ internationales à la politisation explicite et aux engagements militants. Enfin, ils sont souvent sollicités en tant qu'experts (auprès de ministères, de commissions, etc.). L'enquête historique révèle également une tendance à la professionnalisation : les compétences en matière de gestion des problèmes environnementaux, de plus en plus indépendantes du militantisme écologique, sont désormais certifiées par des titres scolaires et/ou par le passage en $\mathrm{ONG}^{25}$. D'autre part, on trouve des chercheurs proches du pouvoir dans ses différentes manifestations (politique, technico-administratif, économique, etc.), que ce soit par leurs rattachements disciplinaires ou institutionnels (instituts de recherche, grandes écoles...). Parfois administrateurs de recherche, ils exercent un pouvoir à la fois symbolique et temporel (direct ou indirect lorsqu'il est média- 
tisé à travers les hommes de pouvoir aux yeux desquels ils font autorité). Porteurs de savoirs traduisibles dans un langage décisionnaire, ils trouvent chez les cadres d'entreprise des interlocuteurs naturels. Si la diversité et la distance en termes d'univers sociaux et de points de vue semble ainsi respectée en de tels lieux, la rencontre entre ces différentes populations n'est pas pure contingence d'une part et, d'autre part, les débats qui s'y jouent exigent pour droit d'entrée un accord a minima - accord qui n'a pas à être explicité entre participants - sur les fondements (les vertus du marché26), les termes (biodiversité, services, utilité, intérêt...) et la forme du débat. À l'échelle micro-sociologique, on voit ainsi que la rationalisation économique du rapport à la biodiversité est le produit d'une dynamique de coproduction qui repose sur la rencontre entre différents groupes sociaux : si ce rapprochement demande efforts et concessions de part et d'autre, il repose également sur des bases solides; et s'il est producteur d'une positivité, il se construit aussi sur des présupposés communs.

\section{LISTE DES ACRONYMES}

AFD : Agence Française de Développement

AFEP : Association Française des Entreprises Privées

CAS : Centre d'Analyse Stratégique

CDB : Convention sur la Diversité Biologique

CIRAD : Centre International de Recherche Agronomique pour le Développement

COP : Conférence des Parties

EPBRS : European Platform for Biodiversity Research

Strategy

EPE : Entreprises pour l'Environnement

FRB : Fondation pour la Recherche sur la Biodiversité

GT : Groupe de Travail

IIED: International Institute for Environment and Development

INRA : Institut National de Recherche Agronomique IPBES : International Science-Policy Platform on Biodiversity and Ecosystem Services

IRD : Institut de Recherche pour le Développement

MEA : Millenium Ecosystem Assesment

MEEDDM : Ministère de l'Écologie, de l'Énergie, du Développement

MIT: Massachusetts Institute of Technology

ONG : Organisation Non-Gouvernementale

PNUE : Programme des Nations Unies pour l'Environnement

SE: Services Écosystémiques

TEEB : The Economics of Ecosystems and Biodiversity

UICN : Union Internationale pour la Conservation de la Nature

WBCSD: World Business Council for Sustainable Development

WRI: World Resource Institute.

WWF: World Wide Fund for Nature 


\section{$R \cdot E ́ \cdot F \cdot E ́(R \cdot E \cdot N \cdot C \cdot E \cdot S$}

AUBERTIN, C., BOISVERT, V., \& PINTON, F. , Les marchés de la biodiversité, IRD, 2007. BLANDIN, P. , De la protection de la nature au pilotage de la biodiversité, Paris, Quae, 2009. BOURDIEU, P. , La noblesse d'État. Grandes écoles et esprit de corps, Paris, Minuit, 1989. BOYD, J., \& BANZHAF, S., What Are Ecosystem Services? The Need for Standardized Environmental Accounting Units, in Resources for the Future, vol. 06, n², 2006.

CONSTANZA, R., "The value of the world's ecosystem services and natural capital", in Nature, n³87, 1997, pp. 253-260.

DAILY, G., Nature's Services : Societal Dependence On Natural Ecosystems, Island Press, 1997. DEZALAY, Y., \& GARTH, B., « Connivence des élites internationalisées », in Le Monde Diplomatique, juin 2005.

DJAMA, M., \& DAVIRON, B. Rationalité managériale et configurations de pouvoir dans les partenariats ONG - Industrie pour le développement durable : le cas de la « Roundtable on Sustainable Palm Oil » (RSPO).

ELIAS, N., La société de cour, Paris, Flammarion, 1993.

FISHER, B., \& KERRY TURNER, R., "Ecosystem services : Classification for valuation" in Biological Conservation, vol. 141, n 5, 2008, pp. 1167-1169

FOUCAULT, M., «Les techniques de soi » (1982), in Dits et écrits, vol. II, Paris, Gallimard, 2001, pp. 1602-1632.

FOUCAULT, M., Naissance de la biopolitique, Cours au collège de France. 1978-1979, Paris, Gallimard, 2004.
GROOT, R. S., "Environmental functions as a unifying concept for ecology and economics", in Environmentalist, nº , 1987, pp. 105-109.

GROOT, R. S., "Functions of Nature : Evaluation of Nature" in Environmental Planning, Management, and Decision Making. Groningen, Wolters-Noordhoff, 1992.

HEAL, G., Nature and the marketplace : capturing the value of ecosystem services, Island Press, 2000.

HOUDET, J., Entreprises, biodiversité et services écosystémiques : Quelles interactions et stratégies? Quelles comptabilités? Thèse de doctorat, AgroParisTech, Paris, 2010.

JEANPIERRE, L., « Une sociologie foucaldienne du néo-libéralisme est-elle possible ? » in Sociologie et sociétés, vol. 38, n², 2006.

KOSOY, N., \& CORBERA, E., "Payments for Ecosystem Services as Commodity Fetishism", in Ecological Economics, ${ }^{\circ} 69,2010$, pp. 1228 1236.

KRIEG-PLANQUE, A., « La formule “développement durable" : un opérateur de neutralisation de la conflictualité » in Langage et société, vol.134, n4, 2010.

LANDELL-MILLS, N., \& PORRAS, I., Silver bullet or fools gold? A global review of markets forest environmental services and their impacts for the poor, London, International Institute for Environment and Development, 2002.

LASCOUMES, P., \& LE GALÈS, P., Gouverner par les instruments, Paris, Presses de SciencesPo, 2004.

LEBARON, F., « La dénégation du pouvoir. Le champ des économistes français au milieu des années 1990 », in Actes de la recherche en sciences sociales, $\mathrm{n}^{\circ} 119$, 1997, pp. 3-26.

LEBARON, F., La croyance économique. Les 
économistes entre science et politique, Paris, Seuil, 2000.

MAHRANE, Y., «L'UICN et son internationalisation ». Intervention au Séminaire « Gouverner le vivant », 2010.

MARIS, V., \& REVÉRÊT, J.-P., « Les limites de l'évaluation économique de la biodiversité ", in Les Ateliers de l'Éthique, n4, 2009, pp. 52-66. MARIS, V., \& REVÉRÊT, J.-P., « L'évaluation économique de la biodiversité et des biens et services écologiques : regards croisés économiques et philosophiques » in Nègre, C. (Ed.), La Convention internationale sur la biodiversité - enjeux et mise en cuvre, La documentation française, 2010, pp. 53-76.

McAFEE, K., "Selling Nature to save it ? Biodiversity and Green developmentalism. Environment and planning", in Society and space, Vol. 2, n'17, 1999, pp. 133-154

MEA. Ecosystems and Human well-being, Washington, Island Press, 2005.

MÉRAL, P., «Les services environnementaux en économie : revue de la littérature ", Document de travail n²010-05, Montpellier, IRD, 2010.

MOONEY, H., \& EHRLICH, P. R., "Ecosystem services : A fragmentary history". in Daily, G. C. (Ed.), Nature's Services. Societal Dependence on Natural Ecosystems, Washington, Island Press, 1997, pp. 11-19.

PAGIOLA, S., LANDELL-MILLS, N., \& BISHOP, J., Selling Forest Environmental Services, Earthscan Publications Ltd, 2002.

TAKACS, D., The idea of biodiversity. Philosophies of Paradise, Baltimore, The Johns Hopkins University Press, 1996.

TRÉPOS, J.-Y., La sociologie de l'expertise, Paris, PUF, 1996.

WALLACE, K. J., “Classification of ecosystem services : Problems and solutions", in Biological Conservation, vol. 3-4, n 139, 2007, pp. 235-246. 
$\mathrm{N} \cdot \mathrm{O} \cdot \mathrm{T} \cdot \mathrm{E} \cdot \mathrm{S}$

1. L'alerte dont il est ici question émane souvent de chercheurs (biologistes, écologues, généticiens...) s'appuyant sur des hypothèses clés - celle de $" 6^{\text {e }}$ extinction » par exemple - dans le but d'interpeller les autorités publiques. L' «Appel des 60 pour la Biodiversité » (adressé en mai 2010 au Président Nicolas Sarkozy par un groupe de chercheurs afin d'exprimer leur inquiétude) en est un parfait exemple. Sur la mise à l'agenda de la biodiversité, consulter D. Takacs, The idea of biodiversity. Philosophies of Paradise, Baltimore, The Johns Hopkins University Press, 1996. 2. Pour une histoire et un état des débats, consulter P. Méral, « Les services environnementaux en économie : revue de la littérature », Document de travail n²010-05, Montpellier, IRD, 2010.

3. Les lignes de fracture entre économistes au sujet de la biodiversité reproduisent probablement celles que l'on observe au sein de l'économie de l'environnement, qui elles-mêmes tendent à reproduire à une échelle réduite les oppositions propres au monde des économistes (F. Lebaron, La croyance économique. Les économistes entre science et politique, Paris, Seuil, 2000). C'est en tous cas ce que laisse à penser l'entrée récente dans la controverse d'une économie d'inspiration marxiste (N. Kosoy \& E. Corbera, « Payments for Ecosystem Services as Commodity Fetishism », in Ecological Economics, ${ }^{\circ}{ }^{\circ}$ 69, 2010, p. 1228-1236) contribuant à cliver les positionnements scientifiques et politiques et donc à rendre plus apparentes les positions qui leurs sont associées. Il ne faut cependant pas oublier qu'à un niveau plus général encore, les tensions se produisent très vraisemblablement entre disciplines ou groupes de disciplines.

4. Signalons tout de même les recherches entreprises par Yannick Mahrane (Centre Koyré, EHESS) au sujet des changements de paradigmes au sein de l'UICN, ainsi que les travaux produits dans le cadre du projet Serena, Services environnementaux et usages de l'espace rural (www.serena-anr.org/).

5. L'article proposé est la présentation provisoire d'une enquête en cours, menée dans le cadre du projet PAN-Bioptique : les institutions émergentes de la biodiversité financé par l'ANR. Il s'appuie plus particulièrement sur un travail sociologique associant : entretiens (avec des chercheurs ou des décideurs), ethnographies, travail sur archives, recherches biographiques et prosopographiques, construction de réseaux et analyses de graphes. Ici, il s'agit surtout de réaliser un rapide état de l'art dans le domaine assorti de pistes de recherche inspirées par notre recherche.

6. «J'appelle « gouvernementalité » la rencontre entre les techniques de domination exercées sur les autres et les techniques de soi » (M. Foucault, «Les techniques de soi ", in Dits et écrits, vol. II, Paris, Gallimard, 2001, p. 1604).

7. Sur tous ces points et pour un exposé plus détaillé, voir L. Jeanpierre, « Une sociologie foucaldienne du néo-libéralisme est-elle possible? » in Sociologie et sociétés, vol. 38, n², 2006.

8. Sur tous ces points, voir Y. Mahrane, L'UICN et son internationalisation. Intervention au Séminaire « Gouverner le vivant », 2010.

9. «Un instrument [...] constitue un dispositif à la fois technique et social qui organise des rapports sociaux spécifiques [...] en fonction des représentations et des significations dont il est porteur. [Les instruments] ne sont pas des outils axiologiquement neutres, et indifféremment disponibles. Ils sont au contraire porteurs de valeurs, nourris d'une interprétation du social et de conceptions précises du mode de régulation envisagé » Lascoumes, P., \& Le Galès, P., Gouverner par les instruments, Paris, Presses de Sciences-Po, 2004, pp. 13-14. 
10. Sur les points suivants, P. Méral, op.cit..

11. Pour une présentation plus détaillée : G. Daily, Nature's Services : Societal Dependence On Natural Ecosystems, Island Press, 1997. On pourrait se demander si les SE ne contribuent pas à la néolibéralisation des pratiques à travers la compétition qu'ils instaurent de fait entre espèces ou écosystèmes, la « mise en concurrence généralisée » étant selon M. Foucault l'eidos de la gouvernementalité néolibérale (M. Foucault, Naissance de la biopolitique, Cours au collège de France. 1978-1979, Paris, Gallimard, 2004).

12. P. Méral, op.cit. et H. Mooney, \& P. R. "Ehrlich, Ecosystem services: A fragmentary history”. In G. C. Daily, (Ed.), Nature's Services. Societal Dependence on Natural Ecosystems, Washington, Island Press, 1997, pp. 11-19.

\section{Idem.}

14. R. S., "Groot, Functions of Nature : Evaluation of Nature" in Environmental Planning, Management, and Decision Making. Groningen, Wolters-Noordhoff, 1992.

15. G. Heal, Nature and the marketplace : capturing the value of ecosystem services, Island Press, 2000 ; N. Landell-Mills, \& I. Porras, Silver bullet or fools gold? A global review of markets forest environmental services and their impacts for the poor, London, International Institute for Environment and Development, 2002 ; S. Pagiola, N. Landell-Mills, \& J. Bishop, Selling Forest Environmental Services, Earthscan Publications Ltd, 2002.

16. http://www.maweb.org/en/index.aspx

17. Maris, V., \& Revérêt, J.-P., « L'évaluation économique de la biodiversité et des biens et services écologiques : regards croisés économiques et philosophiques » In Nègre, C. (Ed.), La Convention internationale sur la biodiversité - enjeux et mise en œuvre, La documentation française, 2010, pp. 53-76. 18. J. Boyd, \& S. Banzhaf, "What Are Ecosystem
Services? The Need for Standardized Environmental Accounting Units", in Resources for the Future, vol. 06, n², 2006. 2006 ; B. Fisher, \& R. Kerry Turner, "Ecosystem services : Classification for valuation" in Biological Conservation, vol. 141, n5, 2008, pp. 1167-1169; V. Maris, \& J.-P. Revérêt, "Les limites de l'évaluation économique de la biodiversité", in Les Ateliers de l'Éthique, n4, 2009, pp. 52-66; K.J. Wallace, "Classification of ecosystem services : Problems and solutions", in Biological Conservation, vol. 3-4, $\mathrm{n}^{\circ} 139,2007$, pp. 235-246.

19. http://www.strategie.gouv.fr/article.php3?id_article $=980$

20. Selon Foucault, les nouveaux types de pouvoir « $n$ 'ont absolument pas pour fonction primordiale de prohiber, d'empêcher, de dire " tu ne dois pas ». La fonction primitive, essentielle et permanente de ces pouvoirs (...) est, en réalité, d'être des producteurs d'une efficience, d'une aptitude, des producteurs d'un produit » (M. Foucault, 2001, op. cit. p.1006).

21. Voir notamment J. Houdet, Entreprises, biodiversité et services écosystémiques: Quelles interactions et stratégies? Quelles comptabilités? Thèse de doctorat, AgroParisTech, Paris, 2010

22. J.-Y. Trépos, La sociologie de l'expertise, Paris, PUF, 1996.

23. Sur les chaînes d'interdépendance, voir N. Elias, $L a$ société de cour, Paris, Flammarion, 1993. Sur l'allongement des chaînes de légitimation, voir P. Bourdieu, La noblesse d'État. Grandes écoles et esprit de corps, Paris, Minuit, 1989.

24. L'analyse de discours révèle que certaines « formules » (parfois dénoncées comme des oxymorons), généralement associées à des concessives, fonctionnent comme des « opérateurs de neutralisation» (KriegPlanque, A., « La formule "développement durable" : un opérateur de neutralisation de la conflictualité » in Langage et société, vol.134, n4, 2010). 
25. Les propriétés spécifiques de cette population de cadres passés par des ONG ont été décrites dans : Y. Dezalay, \& B. Garth, « Connivence des élites internationalisées », in Le Monde Diplomatique, juin 2005 ; M. Djama, \& B. Daviron, Rationalité managériale et configurations de pouvoir dans les partenariats ONG - Industrie pour le développement durable : le cas de la « Roundtable on Sustainable Palm Oil» (RSPO). 26. F. Lebaron, op. cit.

Mots-clés : Biodiversité - Expertise - Dispositif Gouvernance - Néolibéralisme - Rationalisation économique

Keywords: Biodiversity - Expertise - Apparatus - Governance - Neoliberalism - Economic Rationalization

\section{$\mathrm{R} \cdot \dot{E} \cdot \mathrm{S} \cdot \mathrm{U} \cdot \mathrm{M} \cdot \mathrm{E}$}

L' " érosion de la biodiversité » a fait l'objet d'un processus d'alerte et de mise à l'agenda scientifique, politique et médiatique depuis les années 80 . Cette dynamique a conduit à l'émergence d'instances de gouvernance et à l'élaboration de technologies de maîtrise. Parmi celles-ci, le recours aux mécanismes du marché semble l'une des pistes privilégiées et en tout cas des plus discutées. L'objectif du texte suivant est d'une part de rendre compte de la mise en place d'un dispositif institutionnel et instrumental de mise en marché de la biodiversité. D'autre part, il s'agit d'analyser les conditions sociales de production des instruments de rationalisation économique du rapport à la biodiversité et d'apparition d'une communauté de spécialistes.

\section{Abstract}

The "biodiversity loss" has been subjected to a whistleblowing and an agenda-setting process since the 80's (in science, politics and the media). This trend has led to the emergence of governance entities and of management and control technologies. Among these, resorting to the Market has been one of the most salient - or at least one the most discussed - ideas. The aim of the following paper is to describe the appearance of a specific institutional and instrumental apparatus; to analyze the social production of these tools of economic rationalization of the attitude towards biodiversity; and to understand the emergence of a community of specialists. 
